\title{
Heads or tails: cell biological secrets of the spermatozoon revealed
}

\author{
David F. Albertini ${ }^{1}$
}

Published online: 3 February 2016

(C) Springer Science+Business Media New York 2016

Cryobiology has assumed a central place in the daily practice of human ARTs. And despite the expanding utilization of these basic techniques, especially that of vitrification, it is rare for practitioners of reproductive medicine to look beyond how their patients' gametes and embryos have fared after the "big chill" other than to query their survival and quality status before the next scheduled procedure. When it comes to the cryobiological procedures presently in widespread use around the world, how many clinicians or embryologists are aware of the scientists who contributed the foundational work in this field, some of the most prominent of whom have passed in recent years? Among them, the late John Critser was a founding member of the JARG editorial board when I took this position back in 2009, and his vigilance and acumen provided one of the first reports warning about the detrimental effects of high DMSO concentrations on mammalian oocytes, antedating what has since become the business of oocyte banking much to the chagrin of the scientists who forged ahead developing the very protocols now in use today. Both Stan Liebo and Peter Mazur, who recently passed, were also true pioneers in the field of cryobiology as now practiced in human ARTs.

It is our intention at JARG to build on the framework of those scientists whose research careers made possible the translation of solid basic science into clinical platforms at

Capsule Beyond the daily practice of cryobiology in human and ARTs (pl), glimpses of the sperm's motility machinery in the finest of details with cryotomography electron microscopy are reminding us of how defocused we have become with new wave technologies that bring our thinking and practice into the realm of guesswork rather than truth.

David F. Albertini

DALBERTINI@kumc.edu

1 University of Kansas Medical Center, Kansas, KS, USA the heart of infertility treatments such as cryobiology, a topic to be covered in a future special issue. And in keeping with our commitment to educate our readership on the basic biological principles governing human reproduction, we take aim this month at the male germ cell and some of the remarkable properties of the nucleus and motility apparatus that are deepening our understanding of the complexities underlying fertilization and the molecular basis of infertility.

We begin this issue with a comprehensive treatment of the nano-machine that gets sperm where they need to go- the axoneme. Linck and colleagues trace the historical origins of the sperm's motility apparatus and review in some detail how the introduction of electron microscopy revealed the axoneme as an extraordinary device designed to power the movement of sperm and cilia (The axoneme: the propulsive engine of spermatozoa and cilia and associated ciliopathies leading to infertility. J Assist Reprod Genet DOI 10.1007/s10815-016-0652-1). In fact, for those of us who have spent decades in the business of medical education, the discovery that patients with Kartagener syndrome shared a distinctive ultrastructural deficit in their axonemes underscoring the chronic respiratory distress and infertility symptoms with which they were afflicted, stands as the singular and most significant case of "structure and function" association that has tingled the minds of many physicians and scientists in early stages of their training.

The timeliness of this review is further evidenced by coverage of the emerging class of diseases known as ciliopathies, whose genetic and molecular bases are being uncovered in the context of various tissue and organ system pathologies. In this light, it is awe-inspiring to consider that the several hundred genes (by conservative estimates) whose protein products are believed to be required to build the axoneme (and who knows how many post-transcriptional and post-translational steps will be invoked in this construction project that has yet to be teased apart in a mechanistic way) have truly withstood the 
test of evolutionary time as perhaps the most highly conserved macromolecular assembly by which cells move around.

And getting back to cryobiology, it is no accident that our cover this month features a high-resolution three-dimensional rendition of the axoneme of a sea urchin sperm prepared using cryo-tomographic electron microscopy, a technique that allows for evaluation of native structural detail in complex macromolecular aggregates. Interestingly, the group that produced these results has shown that the sea urchin axoneme shown on the cover is virtually identical to that of human respiratory cilia and using this technique have now mapped the defect responsible for primary ciliary dyskinesia in patients with respiratory disease (Lin et al., 2015 Cryo-electron tomography reveals ciliary defects underlying human $\mathrm{RSPH} 1$ primary ciliary dyskinesia. Nat Commun.; 5: 5727. doi:10.1038/ncomms6727). Among the secrets of sperm motility being uncovered by technologies that allow for a penetrating perspective of the axonemal nanomachine, genetic tools are exposing many of the players implicit in understanding causes of male infertility. The studies of Zhang and colleagues report in this issue the identification of polymorphisms in the human tektin-t gene that appear to be linked to idiopathic asthenozoospermia within regions of China (Zhang et. Al., Association of polymorphisms in tektin-t gene with idiopathic asthenozoospermia in Sichuan, China. J Assist Reprod Genet DOI 10.1007/s10815-015-0617-9).

In these dominating years of ICSI, where the motility of human sperm is relegated to the role of chosen tailless (and hence aimless) wonder, it is the compacted genome in the head that finally wins the coin toss for launching embryo development. In the review by Ioannou and collaborators (Impact of sperm DNA chromatin in the clinic. J Assist Reprod Genet DOI 10.1007/s10815-015-0624), the importance of achieving and effecting a degree of chromatin compaction that will be modified by ooplasmic factors to create the new nucleosomal genome required for development to proceed is considered from a clinical perspective. What spermatogenesis accomplishes over the weeks that go into building this remarkable cell, must in the earliest hours after fertilization be completely reversed with the molecular devices the oocyte has acquired during its development and maturation - a matter well known in ART circles to be at the heart of failed fertilization and other interruptions in embryo development prior to or following implantation.

While dissociating heads from tails when it comes to the spermatozoon and its utility in the ART clinic has been a matter of practicality and efficiency for achieving fertilization, the continued pursuit of knowledge at the cell biological and genetic levels must progress through the unionizing of new technologies that provide complementary insights into the causes and malignments of gamete physiology at the heart of human infertility. As reproductive medicine and biology move forward for the betterment of society, it is important to bear in mind that the temptation to adopt nouveau technology is strong when commercial incentives abound. Hopefully, new wave technology will also have a place in the discovery of biological principles yet to be applied to the practice of human ARTs. 